\title{
STABLE HARMONIC 2-SPHERES IN SYMMETRIC SPACES
}

\author{
F. BURSTALL, J. RAWNSLEY AND S. SALAMON
}

A map $\phi:(M, g) \rightarrow(N, h)$ of Riemannian manifolds is harmonic if it extremizes the energy $E: C^{\infty}(M, N) \rightarrow R$ given (for compact $M$ ) by

$$
E(\phi)=\frac{1}{2} \int_{M}|d \phi|^{2} \text { vol. }
$$

A harmonic map $\phi$ is said to be stable if the second variation of $E$ at $\phi$ is positive semidefinite. That is: for all smooth variations $\phi_{t} \in C^{\infty}(M, N)$ with $\phi_{0}=\phi$ we have

$$
d^{2} /\left.d t^{2} E\left(\phi_{t}\right)\right|_{t=0} \geq 0 .
$$

Of particular interest is the case where $M$ is the sphere $S^{2}$ and $N$ is a Riemannian symmetric space $G / K$. In this setting harmonic maps are branched minimal immersions, or the finite action solutions of the Euclidean nonlinear $\sigma$-model studied by physicists (see e.g. [20] and references cited therein). In the case $G / K$ is Hermitian symmetric it follows from an argument of Lichnerowicz [9] that any holomorphic map is energy minimizing in its homotopy class and hence stable. The same is true of antiholomorphic (or-holomorphic) maps. The tholomorphic maps are the instantons of the nonlinear $\sigma$-model, and it is important to know if these are the only stable solutions. This is clearly not the case, as one sees by taking $G / K$ to be a product of Hermitian symmetric spaces and by taking a map which is holomorphic into one factor and -holomorphic into the other. However, this is the only way a stable map can fail to be \pm holomorphic, as the following theorem shows.

THEOREM 1. Let $\phi: S^{2} \rightarrow G / K$ be a stable harmonic map into an irreducible Hermitian symmetric space. Then $\phi$ is tholomorphic.

This generalizes a result of Siu and Yau [16], who obtained Theorem 1 for the complex projective spaces as targets.

If the target $G / K$ is a general symmetric space $\phi$ can always be lifted to a map into the simply connected covering space. A simply connected symmetric space then splits as a product of irreducible spaces with $\phi$ given by a harmonic map into each factor. As noncompact factors have nonpositive curvature the component of $\phi$ going into such a factor must be constant (by the results of Eells and Sampson [2], or more simply by the maximum principle [4]), which reduces us to the consideration of compact irreducible symmetric spaces. Moreover $\phi$ is stable if and only if all its components are. We can show that stable harmonic maps into irreducible compact Riemannian symmetric

Received by the editors July 29, 1986.

1980 Mathematics Subject Classification (1985 Revision). Primary 58E20; Secondary $53 \mathrm{C} 35,53 \mathrm{C} 42$. 
spaces always factorize through certain immersed totally geodesic irreducible Hermitian symmetric subspaces. This gives

THEOREM 2. Let $\phi: S^{2} \rightarrow G / K$ be a nonconstant stable harmonic map into any Riemannian symmetric space. Then there is a Hermitian symmetric space $G_{1} / K_{1}$ totally geodesically immersed in $G / K$ such that $\phi$ factorizes through $G_{1} / K_{1}$ as a holomorphic map. Moreover any holomorphic map of a Riemann surface into $G_{1} / K_{1}$ gives a stable harmonic map into $G / K$.

The spaces $G_{1} / K_{1}$ are compatible with the decomposition of $G / K$ into irreducible factors, and are irreducible when $G / K$ is. Although there are different submanifolds and complex structures $G_{1} / K_{1}$ for different maps, modulo the action of the isometry group $G$, there are only a finite number of possibilities for $G_{1} / K_{1}$ and these can be read off the extended Dynkin diagram of $G$. In fact when $G / K$ is irreducible and not itself Hermitian symmetric, the subspaces $G_{1} / K_{1}$ all turn out to be complex projective spaces.

Compact irreducible semisimple symmetric spaces can be broken into three classes according to their second homotopy groups $\pi_{2}(G / K)$ : we know the Hermitian symmetric spaces are characterized completely by $\pi_{2}(G / K)=Z$. The remaining two possibilities are $\pi_{2}(G / K)=0$ (this includes all the Lie groups viewed as symmetric spaces) or $\pi_{2}(G / K)=Z_{2}$. In the first case there are never any of the Hermitian symmetric subspaces of the $G_{1} / K_{1}$ type available and so we have

THEOREM 3. Let $\phi: S^{2} \rightarrow G / K$ be a stable harmonic map into a symmetric space with $\pi_{2}(G / K)=0$. Then $\phi$ is constant.

In the remaining case where $\pi_{2}(G / K)=Z_{2}$, when $G / K$ is irreducible the various possible inclusions $G_{1} / K_{1} \subset G / K$ induce surjections on $\pi_{2}$, so we obtain

THEOREM 4. If $G / K$ is a symmetric space then every homotopy class of maps $S^{2} \rightarrow G / K$ has a stable harmonic representative.

REMARKS. 1. The irreducible symmetric spaces with $\pi_{2}(G / K)=Z_{2}$ are $\mathrm{SU}(n) / \mathrm{SO}(n), n \geq 3, \mathrm{SO}(p+q) / \mathrm{SO}(p) \times \mathrm{SO}(q), p \geq q \geq 3$, together with the following exceptional spaces, which we list along with the largest of the $G_{1} / K_{1}$ subspaces for each:

$$
\begin{aligned}
& E_{6} / \mathrm{Sp}(4)^{\sim}, \mathrm{CP}^{2} ; \quad E_{6} / \mathrm{SU}(6) \mathrm{Sp}(1), \mathrm{CP}^{4} ; \quad E_{7} / \mathrm{SU}(8)^{\sim}, \mathrm{CP}^{5} ; \\
& E_{7} / \mathrm{Spin}(12) \mathrm{Sp}(1), \mathrm{CP}^{6} ; \quad E_{8} / \mathrm{Spin}(16)^{\sim}, \mathrm{CP}^{8} ; \quad E_{8} / E_{7} \mathrm{Sp}(1), \mathrm{CP}^{7} ; \\
& F_{4} / \mathrm{Sp}(3) \operatorname{Sp}(1), \mathrm{CP}^{2} ; \quad G_{2} / \mathrm{SO}(4), \mathrm{CP}^{2} .
\end{aligned}
$$

Here $K^{\sim}$ denotes a quotient $K / Z_{2}$. The spaces in this list where $K$ is not simple are the quaternionic Kähler manifolds studied by Wolf [19].

2. Sacks and Uhlenbeck [14] show by general methods that $\pi_{2}(G / K)$ has a set of stable harmonic generators; we can exhibit explicit generators which are embedded homogeneous 2-spheres arising from suitably chosen root spaces. When $\pi_{2}(G / K)=Z_{2}$ we can precompose such stable harmonic maps with holomorphic maps of $S^{2}$ of arbitrarily high degree without losing stability and 
so represent the trivial and nontrivial homotopy classes by nonconstant stable harmonic maps of arbitrarily high energy.

3. The result of Theorem 3 on the nonexistence of nonconstant stable harmonic maps follows by case-by-case checking from the work of Howard and Wei [6], Ohnita [12], Tyrin [18] (for the classical spaces), and Smith [17], since the list of irreducible spaces (of type III) with $\pi_{2}(G / K)=0$ coincides with the list

$$
S^{n}, \quad \mathrm{SU}(2 n) / \mathrm{Sp}(n), \quad \mathrm{Sp}(p+q) / \mathrm{Sp}(p) \times \operatorname{Sp}(q), \quad E_{6} / F_{4}, \quad F_{4} / \operatorname{Spin}(9)
$$

of spaces with unstable identity maps. However the Lie groups with stable identity maps still have no nonconstant harmonic 2-spheres as a consequence of Theorem 3. This is also a consequence of a construction by Uhlenbeck of a canonical energy-decreasing variation of a nonconstant harmonic map. See also Pluzhnikov [13] for related results on the instability of the identity map.

4. Micallef [10] has recently shown the existence of nonconstant harmonic maps $S^{2} \rightarrow G$ of index $\leq 1$. As a consequence of Theorem 3 these maps are seen to have index precisely equal to 1 .

5. For maps of $S^{2}$ the index of the energy agrees with the index of the area, so all the above theorems apply with harmonic maps replaced by minimal 2spheres.

6. Our method depends in an essential way on the structure theorem of Grothendieck [5] concerning holomorphic bundles over the Riemann sphere. For Riemann surfaces of higher genus we can obtain the results of Siu [15] and Leung [8] for complex projective spaces or $n$-spheres respectively, with the same restrictions on the branching of the map. By using a twistor space over the domain manifold, Burns and De Bartolomeis have recently shown that all harmonic maps of a Riemann surface of any genus into a complex projective space must be \pm holomorphic.

7. The index of unstable maps is not known in general, but Ejiri [3] has shown that any full harmonic map from $S^{2}$ to $S^{2 n}$ has index at least $2 n(n+2)-6$.

SKETCH OF THE PROOFS. We identify the tangent bundle to $G / K$ with the orthogonal $[\mathfrak{p}]$ of the bundle $[\mathfrak{k}]$ of Lie algebras of the stability subgroups in the trivial bundle $\mathfrak{g}$. The Levi-Civita connection in $[\mathfrak{p}]$ induces a connection in $[\mathfrak{k}]$ and so the direct sum connection in $\mathfrak{g}$. This may be pulled back to $S^{2}$ by a map $\phi: S^{2} \rightarrow G / K$ to give a connection $\nabla^{\phi}$ in the trivial bundle $\mathfrak{g}$ of Lie algebras over $S^{2}$. The theorem of Koszul and Malgrange [7] gives the complexification $\mathfrak{g}^{c}$ the structure of a holomorphic bundle of Lie algebras with the $(0,1)$ part of $\nabla^{\phi}$ being the $\bar{\partial}$-operator. Grothendieck's structure theory [5] tells us this bundle contains a holomorphic bundle of parabolic subalgebras $q$ compatible with the decomposition $\mathfrak{g}^{c}=\phi^{-1}[\mathfrak{p}]^{c}+\phi^{-1}[\mathfrak{k}]^{c}$. This bundle of parabolics can be modified as in [1] so that its nilradical bundle $\mathfrak{n}$ is generated by its intersection with $\phi^{-1}[\mathfrak{p}]^{c}$ and this intersection is contained in the subbundle of $\phi^{-1}[\mathfrak{p}]^{c}$ generated by holomorphic sections. On the other hand Moore [11] gives the Hessian of the energy as

$$
\operatorname{Hess}(u, v)=4 \int_{S^{2}}\left(\nabla_{\bar{z}}^{\phi} u, \nabla_{z}^{\phi} v\right)-([\delta, u],[\bar{\delta}, v]) i / 2 d z \wedge d \bar{z}
$$


where $u$ and $v$ are sections of $\phi^{-1}[\mathfrak{p}]^{c}$ and $\delta$ is $\phi * \partial / \partial z$ viewed as a section of $\mathfrak{G}^{c}$. Thus if $\phi$ is stable, taking $\nabla_{\bar{z}}^{\phi} u=0$ we see that $\delta$ must commute with all holomorphic sections of $\phi^{-1}[\mathfrak{p}]^{c}$ and hence with the nilradical bundle $\mathfrak{n}$. But $\delta$ takes its values in $\mathfrak{n}$, and hence must be in the center $\mathfrak{z}$ of $\mathfrak{n}$. A Lie-theoretic argument shows that when $G$ is simple any parabolic has an irreducible action on the center of its nilradical. If $G / K$ is Hermitian symmetric the parabolic is compatible with the complex structure and so $z$ must be contained in either the $(1,0)$ or $(0,1)$ vectors giving Theorem 1 . Theorem 2 follows by observing that $z+\bar{z}$ gives rise to a Lie triple system such that $z$ is tangent to an immersed totally geodesic Hermitian symmetric subspace $G_{1} / K_{1}$ through which $\phi$ can be shown to factorize holomorphically as a consequence of the vanishing of certain holomorphic differentials. The Dynkin diagram of $G_{1}$ is obtained from the extended Dynkin diagram of $G$ by striking out the complementary simple roots of $\mathfrak{q}$ and taking the connected component of the negative of the highest root in what remains. Theorem 3 is obtained by showing that when $\pi_{2}(G / K)=0, z$ is always in the stability subalgebra. This forces $\delta$ to vanish and so $\phi$ is constant. Theorem 4 also follows since we can describe the $G_{1} / K_{1}$ subspaces so explicitly.

ACKNOWLEDGMENT. It is a pleasure to thank Jim Eells for his help and advice and John C. Wood for bringing reference [18] to our attention.

\section{REFERENCES}

1. F. Burstall and J. Rawnsley, Twistors, harmonic maps and homogeneous geometry (to appear).

2. J. Eells and J. Sampson, Harmonic mappings of Riemannian manifolds, Amer. J. Math. 86 (1964), 109-160.

3. N. Ejiri, The index of minimal immersions of $S^{2}$ into $S^{2 n}$, Math. Z. 184 (1983), 127-132.

4. W. B. Gordon, Convex functions and harmonic maps, Proc. Amer. Math. Soc. 33 (1972), 433-437.

5. A. Grothendieck, Sur la classification des fibrés holomorphes sur la sphère de Riemann, Amer. J. Math. 79 (1957), 121-138.

6. R. Howard and S. W. Wei, Non-existence of stable harmonic maps to and from certain homogeneous spaces and submanifolds of Euclidean space, Trans. Amer. Math. Soc. 294 (1986), 319-331.

7. J. L. Koszul and B. Malgrange, Sur certaines structures fibrés complexes, Arch. Math. 9 (1958), 102-109.

8. P. F. Leung, On the stability of harmonic maps, Lecture Notes in Math., vol. 949, Springer-Verlag, Berlin and New York, 1982, pp. 122-129.

9. A. Lichnerowicz, Applications harmoniques et variétés Kähleriennes, Symposia Math. 3 (1970), 341-402.

10. M. J. Micallef, On the topology of positively curved manifolds (preprint).

11. J. D. Moore, Compact Riemannian manifolds with positive curvature operators, Bull. Amer. Math. Soc. 14 (N.S.) (1986), 279-282.

12. Y. Ohnita, Stability of harmonic maps and standard minimal immersions, Tôhoku Math. J. 38 (1986), 259-267.

13. A. Pluzhnikov, On the minimum of the Dirichlet functional (to appear) (Russian)

14. J. Sacks and K. Uhlenbeck, The existence of minimal immersions of 2-spheres, Ann. of Math. (2) 113 (1981), 1-24.

15. Y.-T. Siu, Some remarks on the complex-analyticity of harmonic maps, Southeast Asian Bull. Math. 3 (1979), 240-253. 
16. Y.-T. Siu and S.-T. Yau, Compact Kähler manifolds of positive bisectional curvature, Invent. Math. 59 (1980), 189-204.

17. R. T. Smith, The second variation formula for harmonic mappings, Proc. Amer. Math. Soc. 47 (1975), 229-236.

18. A. V. Tyrin, Critical points of the multidimensional Dirichlet functional, Mat. Sb. 124 (1984), 146-158. (Russian)

19. J. A. Wolf, Complex homogeneous contact manifolds and quaternionic symmetric spaces, J. Math. Mech. 14 (1965), 1033-1047.

20. W. Zakrzewski, Classical solutions of two-dimensional Grassmannian models, J. Geom. Phys. 1 (1984), 39-63.

Department of Mathematics, UniVersity of BAth, Bath BA2 7AY, ENGLAND

Mathematics Institute, UNIVERSity of WARWICK, COVENTRY CV4 7AL, ENGLAND

Mathematical institute, University of OXford, 24 St. Giles, OXford OX1 3LB, ENGLAND 